\title{
Automatic quality control of digitally reconstructed radiograph computation and comparison with standard methods
}

\author{
Eloïse Denis ${ }^{a}$, Stéphane Beaumont ${ }^{b}$, JeanPierre Guédon ${ }^{a}$, \\ Nicolas Normand ${ }^{a}$ and Tarraf Torfeh ${ }^{a}$ \\ ${ }^{a}$ IRCCyN/IVC - UMR CNRS 6597, Image \& Video Communication, \\ Ecole polytechnique de l'Université de Nantes, \\ La Chantrerie, BP 50609, 44306 Nantes Cedex 3 - France; \\ ${ }^{b}$ QualiFormeD Sarl - Centre Hospitalier Départemental 85925 La Roche Sur Yon - France
}

\begin{abstract}
Conformal radiotherapy helps to deliver an accurate and effective cancer treatment by exactly targeting the tumor. In this purpose, softwares of the treatment planning system (TPS) compute every geometric parameters of the treatment. It is essential to control the quality of them because the TPS performances are directly connected with the precision on the treated region. The standard method to control them is to use physical test objects (PTOs). ${ }^{1,2}$ The use of PTOs introduces uncertainties in the quality assessment because of the CT scan. Another method to assess the quality of these softwares is to use digital test objects (DTOs). ${ }^{3-5}$ DTOs are exactly known in a continuous and a discrete way. Thus the assessment of the TPS quality can be more accurate and faster. The fact that the DTO characteristics are well known allows to calculate a theoretical result. The comparison of the TPS and this theoretical results leads to a quantitative assessment of the TPS softwares quality. This work presents the control of major quality criteria of digitally reconstructed radiograph (DRR) computation: ray divergence, ray incidence and spatial resolution. Fully automated methods to control these points have been developed. The same criteria have been tested with PTO and the quality assessments by the two methods have been compared. The DTO methods appeared to be much more accurate because computable.
\end{abstract}

Keywords: Quality control, DRR, TPS, CT-simulation, DTO, PTO

\section{INTRODUCTION}

Conformal radiotherapy helps to deliver an accurate and effective treatment against cancer by exactly targeting the tumor. CT-simulation allows a geometrical conformation of the treatment beams and a dosimetric study of the treatment. The geometrical conformation is done in several steps consisting in image registration from different modalities, manual or automatic tumor contouring, automatic target expansion, isocenter computation, beams parameterization (incidence angles, shapes, dimensions and collimator rotation), DRR computation and skin marking for pre-positioning. All these steps are manual, semi-manual or automatic. They are managed by the Treatment Planning System (TPS) which presents many tools to help the radiotherapist to plan the treatment. All these softwares included in the TPS have to be controlled with meticulous care because they are guaranteeing the accuracy of the treatment. Some physical tools for CT-simulation quality control have been developed but they introduce some errors in the quality assessment by their physical nature. From that consideration, a new method of quality assessment for radiotherapy TPS has been developed. This new method is based on digital test objects (DTO). The principle of these objects has been validated ${ }^{3}$ for TPS quality control. One of the advantages of DTO is their flexibility. ${ }^{4}$ A software to develop DTO with a discrete and a continuous description equivalence has been developed. Generated DTOs can be used to control the quality of the TPS like physical test objects (PTOs) but as they are designed specially, it is thus possible to develop

Further author information: (Send correspondence to Eloïse Denis.)

$a$ E-mail: \{eloise.denis, jean-pierre.guedon, nicolas.normand, tarraf.torfeh\}@polytech.univ-nantes.fr

a E-mail: stephane.beaumont@qualiformed.com

Medical Imaging 2007: Physics of Medical Imaging, edited by Jiang Hsieh, Michael J. Flynn, Proc. of SPIE Vol. 6510, 65104J, (2007) · 1605-7422/07/\$18 · doi: 10.1117/12.708193 
an automatic and quantitative method to analyze the TPS results. DTO shapes and analyzing method are developed simultaneously, that improves their efficiency. The shape is well known and not damaged by the scanner acquisition. It is thus possible to compute a theoretical result the TPS should give if it were perfect and without default. The effective result of the TPS is compared with this theoretical result and a qualitative and quantitative assessment of the quality can be done.

This new method is compared to the classical one focuses on three criteria for DRR quality control: beam divergence, beam incidence and spatial resolution. Firstly the classical quality control methods are exposed for these three criteria, then the new methods to control them are presented and finally, the results of a TPS quality assessment by the two methods are shown and compared.

\section{STATE OF THE ART}

The TPS quality control of the geometric tools of the TPS is traditionally done using physical test objects put on the scanner acquisition table like a regular patient. ${ }^{6}$ Particularly two phantoms are used in DRR quality assessment. The first one is used for beam incidence and beam divergence criteria. ${ }^{1}$ The second one is used to control high and low contrast resolution and beam linearity. ${ }^{2}$

Principal tests to carry out for a DRR computation quality control are spatial resolution, contrast, beam incidence and beam divergence. This list is not exhaustive but only identifies the principal quality criteria to check. This section presents the state of the art for the quality control of these criteria.

\subsection{Beam divergence quality control}

Beam divergence in DRR computation needs to be controlled. Indeed, the American Association of Physicists (AAPM) advises in its task group $n^{\circ} 53$ on radiotherapy ${ }^{7}$ to check the correctness of beam divergence in DRR computation. In his task group $n^{\circ} 66$ on CT-simulation, ${ }^{6}$ the AAPM advises to use Craig ${ }^{1}$ and McGee $^{2}$ phantoms to control this criterion. They are two physical test objects that need to be lain on the acquisition table and to be scanned. The three dimensional virtual model of each phantom is then sent to the TPS like a normal patient. These two phantoms will be described, as well as others designed on the study of the two first PTOs.

Craig's phantom ${ }^{1}$ is made up of two acrylic volumes and allows a quality control for the whole TPS. The object used for beam divergence control in DRR computation is a acrylic, cedar and polystyrene bloc with a divergent shape. The different materials delimit the geometry of a divergent irradiation beam for fields with different side sizes. The isocenter is marked by a one millimeter diameter tungsten ball. This divergent shape is inserted in a frame and a basis, which ensures two degrees of freedom. This allows several rotations of the shape in order to place it in the axis of the tested beam. The two degrees of freedom simulate the rotation of the treatment table and the gantry. This object was designed in order to check the beam geometry and can then be used for DRR computation control. The phantom is acquired in the desired position (chosen couch and gantry angles and object correctly aligned with the lasers) and the virtual model is sent to the TPS. The physician is then led to create a new beam which orientation matches with the chosen angles for acquisition positioning and to compute the corresponding DRR. The divergent shape is made in a way that if the source is correctly positioned (at the source to skin distance specified by the manufacturer), it follows the beam divergence and its projection onto the DRR must present neat outlines. Divergence quality control of this resulted DRR consists on determining if the object outlines are neat or not. To do that, the physician needs to compute some other DRRs of beams with one or two degrees more or less from the theoretical angle values, and to evaluate which DRR is the neatest. It is thus not very easy to determine divergence quality of the DRR computation with this method. Slices thickness and acquisition diameter are moreover parameters that make the assessment more or less easy to carry out. Indeed, slices thickness is usually 1 millimeter and slice pixel size is usually about 0.25 millimeter. The spatial resolution of the virtual model is thus different along the three different axis. It is four times finer along $\mathrm{X}$ and $\mathrm{Y}$ axis than along $\mathrm{Z}$ axis. In DRR projection image, outlines perpendicular to the $\mathrm{Y}$ axis are blurrer than the other ones. A commercial version named Quasar of this test object have been developed by Modus. ${ }^{8,9}$ It is thus more difficult to evaluate the sharpness of the divergent shape projection on the DRR image. Divergence quality control with the recommended method is thus dependent on slice thickness and object positioning accuracy on the CT scan table. Moreover, the quality assessment of the DRR is subjective. By the 
physical nature of the object, although it is possible to test many couch and gantry angles, it is impossible to test the divergence for other field sizes, or source to skin distances.

McGee test object $^{2}$ was designed exclusively for DRR computation quality control. It is made up of a acrylic cube of $15 \mathrm{~cm}$ side with several patterns on each face allowing quality assessment for different DRR generation quality criteria verification. Thus it presents a part to control the beam divergence. The corresponding pattern is made up of a brass wire ( 1 millimeter diameter) of 1.5 centimeters long and encrusted on the superior face of the cube, and of two other identical wires encrusted on the opposite face. The first one is placed at 5 centimeters from the central axis of the cube while the other are respectively at 5.38 and 6.25 centimeters from the axis. These positions have been chosen in order to obtain a superimposing of two wires onto the DRR for a source to skin distance of 20 centimeters and 60 centimeters respectively. In the same way as every physical test object using, McGee phantom has to be scanned and the resulted virtual model has to be sent to the TPS. The physician has then to compute DRRs for the two indicated source to skin distances and check that the patterns are superimposed on each image. If they are not, it is thus possible to measure the gap with the graphical tools of the TPS and then calculate the error. This manipulation is not very easy to carry out because of the precision of the tool and is not very accurate. This object allows to check the divergence for two source to skin distances but for only one incidence direction and one beam field size.

Medical physics unity of Tenon hospital in Paris developed its proper phantom for TPS quality control, and thus for DRR quality control. ${ }^{10,11}$ Only one object allows the quality assessment for the whole TPS. It is a polystyrene cube of $20 \mathrm{~cm}$ side. The principle to assess divergence quality is the same as the McGee one, that is to say to check the good superimposition of two patterns on the DRR. A 6 centimeter diameter circle is engraved on the top face of the cube and two circles of respectively 6.6 and 8 centimeters are engraved on the opposite face. Like for the McGee phantom, these patterns allow to control the good divergence of the beam for source to skin distances of 60 and 200 centimeters. As it is as well a physical test object, the quality assessment process is the same as for the McGee test object.

Barry O'Connell ${ }^{12}$ carried out a comparative study of DRR computation quality on two different TPSs (Varian Eclipse and Medcom ProSoma), with the help of a home made phantom based on the McGee one. For the divergence test, the two test objects are similar. Used materials and dimensions are the same. During the DRR divergence quality control, it appeared that the patterns were not aligned on the resulted DRR of the two tested TPSs. The gaps between the pattern images were measured with the graphical tools of each system. The ProSoma ruler was not enough precise and did not allow to measure a such small distance. Thus only Eclipse could really be tested. They concluded that this McGee method is thus not adapted to the DRR divergence control of every TPS. It is possible to see if the divergence is correct on each one but it is not possible to measure the possible error. In this study, some DRR divergence errors have been detected. However, it was proved that it was not because of a ray tracing algorithm weakness but because of a bad positioning of the physical object on the scan table before the acquisition.

McGee method is thus not optimal for the DRR divergence checking. The physical nature of the test object makes the quality control dependent on the physician precision, on the object positioning and on the use of the graphical tools onto the resulted image. Moreover, the measure of the error is limited by the accuracy of the measure tools of the TPS.

Nicholas A. Mason ${ }^{5}$ the first to our knowledge to suggest a digital test object to control TPS algorithms for DRR computation. During his Phd, he developed a software allowing DTO definition and generation. This work was illustrated by the creation of DTOs for DRR beam divergence quality control. The solution he implemented is based on the Craig phantom. The principle is to symbolize the four corners of the beam field by four digital straight lines, and the center of the field by a fifth line. The lines are defined in a continuous way and are rotated in order to fit the chosen divergence. Then, they are rotated again according to two rotation angles in order to simulate the beam incidence determined by the gantry and the couch angles. The continuous volume obtained is then discretized and makes the virtual model to send to the TPS. If the divergence is perfect in the DRR computation, each line is projected in a single dot on the resulted image and the DRR presents four dots for the corners of the beam and one for its center. If it is not perfect, the lines appear as segments on the image that can be measured. This method does not use the scan as the ones based on physical test objects. Thus, it does not involve all the problems due to the object positioning and acquisition. On the other hand, the results 
analysis is still subjective. This software method allows to test every source to skin distances, incidence angles or field sizes because it does not depend on a manufactured object.

\subsection{Beam incidence quality control}

The AAPM task group $n^{\circ} 53^{7}$ mentions the points to check for a good use of the TPSs. It thus recommends to check the orientation of the beams in the DRR computation. In its task group $n^{\circ} 66,{ }^{6}$ the American Association of Physicists advises the use of the Craig object test to control the quality of the beam incidence of the DRR computation algorithms.

The Craig test object was designed to control the whole beam geometry in the CT-simulation. The rotative object described above thus allows to control the incidence of the beams. Although more conceived to control the three dimensional geometry view, this phantom is also advised for the control of the DRRs. Its use is in this case is similar with that for the divergence control. The phantom is positioned on the table of the scan in the position corresponding to the angle of incidence that the physician wants to test, and then its virtual model is transferred to the TPS. Once the corresponding DRR is calculated, the physician has to check if the outlines are neat and then to compare the clearness of the DRR with gantry and couch angles corresponding to those applied to the physical object for the acquisition and that of some DRRs shifted of a few degrees. The clearest one will provide the error of incidence of the DRR calculation. The assessment of the clearness of the outlines on the produced image is not always obvious. The thickness and spacing of the slices, as well as the size of the field of acquisition are such factors which contribute to make the image more or less blurred, as it had been explained in the section relating to the quality control of the beam divergence. A divergence quality assessment with this phantom is very time consuming. For each tested incidence, it is necessary to turn precisely the phantom around the two rotation axis (representing couch and gantry rotations) and make a new scanner acquisition. To carry out a good incidence test, it is important to control each rotation by steps of ten degrees. The table turns from $-90^{\circ}$ to $90^{\circ}$, that makes 18 angles to test and the gantry turns from $0^{\circ}$ to $360^{\circ}$, that makes 36 angles to test. Finally, it is necessary to check the incidence for 648 different angles and consequently to make as many scanner acquisitions.

The McGee physical phantom ${ }^{2}$ comprises a part making it possible to test the beam incidence. One of its faces includes a grid allowing to check the space linearity of the DRR. Some of the graduations (one in each of the four directions) are rotated of $45^{\circ}$. This thus makes possible to control the rotation of the couch and the collimator, by step of $45^{\circ}$. Indeed, after having scanned the phantom and transferred it to the TPS, DRRs with couch and collimator angles multiples of $45^{\circ}$ can be computed. The turned graduations are separated by 10 centimeters that means that they are placed on each corner of an image of a $7.5 \mathrm{~cm}$ field beam if the incidence is correct. It is also possible to check the virtual rotation of the gantry by another handling. Like the majority of the other patterns of this phantom, the grid for the control of the space linearity is made of brass wires of $1,02 \mathrm{~mm}$ diameter. With this phantom the tests are limited to some angles because there is no possibility to turn it.

Mason illustrated his work on a DTO creation software by reproducing digitally a simple Craig phantom and can then control the beam incidence in DRR computation. The DTO is the same that the one for the divergence test. In the same way, if the incidence is correct, five dots appear on the image, unless, it is five small lines. Mason gets then one DTO for one tested incidence angle, in the same way that with the Craig PTO, there are as many series of slices as of tested angles. Mason tested about ten incidences that is to say a combination of angles of $0,45,90$ and 88 degrees for the couch and the gantry. Some observers evaluated the resulted DRR images and determined the smallest detectable error to be 0.2 degree. The assessment of the results is not made in an automatic way. The blur around the dots is measured by counting visually the number of affected pixels by the dot on the DRR. It is sometimes difficult to count these pixels on certain TPSs. Mason work was carried out within the context of the development of a TPS. The tests carried out made it possible to improve and validate the performances of this TPS.

Reilly developed an automatic quality assessment software for TPS from CT-data of physical phantoms. ${ }^{13}$ He began in the same time to design a physical phantom for DRR quality control. This PTO has a tetrahedron shape and allows a verification of the incidence for a null angle of the couch and the gantry. However, the development of this phantom was not finished at the time of its presentation. ${ }^{13}$ 


\subsection{Spatial resolution quality control}

Spatial resolution is an important criterion to control. The $\mathrm{AAPM}^{6}$ recommends to use the McGee phantom ${ }^{2}$ to carry out DRR spatial resolution assessment. Spatial resolution depends on many factors like slice thickness or reconstruction field of view size. Manufacturers do not give DRR spatial resolution, each hospital has to measure it.

McGee PTO is designed to control exclusively DRR computation quality and presents a resolution test. This test is made up of a series of five line pair patterns which frequencies are ranged from 0.1 to 0.5 line pair per millimeter. When a resolution control is carried out with this PTO, spatial resolution depends on the number of pixels on the DRR, the size of the reconstruction field, the slices thickness, the pixel size of the slices and the chosen algorithm to compute the DRR (nearest neighbors, Siddon, etc.). The test patterns are placed parallel to the scan slices plan in order to see the influence of acquisition slice thickness and spacing on the DRR spatial resolution. A practical approach of the MTF (Modulation Transfer Function) is calculated using Droege formula. ${ }^{14}$

Droege defined a method to calculate scans practical Modulation Transfer Function $\left(M T F_{p}\right)$ from a series of line pair patterns encrusted in a test object. This method can be adapted to the quality control of DRR computation. This calculates the $M T F_{p}$ only for the frequencies of the test patterns and not the whole $M T F_{p}$. This method is based on a standard deviations study in the image of area corresponding to the patterns. Thus the ${ }_{p} M T F$ value of a DRR generation system at a frequency $f$ can be obtained from the following formula adapted from the Droege one:

$$
M T F_{p}(f)=\frac{\pi}{\sqrt{2}} \frac{\sqrt{M^{2}-N^{2}}}{\left|D R R_{1}-D R R_{2}\right|}, \quad f>\frac{f_{c}}{3},
$$

where $f_{c}$ is the cutoff frequency, $M$ is the standard deviation of pixel values within the pattern area, $N$ is the standard deviation of a uniform area of the image, $D R R_{1}$ is the mean pixel value of an area corresponding to a part of the phantom which thickness is the same as the one where the patterns are (in the space part of the pattern), $D R R_{2}$ is the mean pixel value of the DRR within an area corresponding to a part of the phantom which thickness is the same as the one where the patterns are (in the plain part of the pattern).

Droege defined a method to evaluate the practical $M T F_{p}$ that is used by many. However, Kirwin preferred to estimate directly the MTF with a Driac signal. ${ }^{15}$ She used for that purpose a physical phantom that is a wax cube with a tungsten ball of 1 millimeter diameter inside. The small ball with a high density is the best way to simulate a Dirac input. It is small enough to be assimilated to a point and big enough to be visible on the DRR. The MTF is then the two dimensional Fourier transform of the ball image (that is the PSF: Point Spread Function). With this method too, a direct relationship is established between slice thickness of CT-data and the DRR computation MTF. Droege formula is applicable for the cases where the frequency is higher than the third of the cutoff frequency. That can ask for very fine line pair patterns that can be difficult to see on the DRR when the CT slices are a bit thick (scanner deteriorates the test patterns). Kirwin carried out some tests with her method and showed that the thicker the slices are, worse the DRR computation MTF is.

The MTF computed by the described method is not really the DRR computation MTF but the whole system scan plus DRR computation MTF. However, if the scan MTF is statistically known, it is possible to recover the specific one to the DRR algorithm from the following relation:

$$
M T F_{\text {system }}(f)=M T F_{\text {scan }}(f) \times M T F_{D R R a l g o r i t h m}(f) .
$$

The interest of these methods of spatial resolution assessment is that their analysis can rather easily be automated. Andrew Reilly developed a software allowing TPS quality control automated analysis and specially scan OTP automated analysis. ${ }^{13}$ It is rather difficult to analyze automatically an integrated system because the provided images are not obviously adapted to control. This software was evaluated ${ }^{16}$ with the use of classical physical test object as the Catphan. The results proved that this automatic analysis is reliable and that the quality control of the scan was much faster than with the manual analysis of the results. Indeed, it took only five minutes against the two required hours for a standard control. However, the Reilly method requires a physical test object and thus all the disadvantages of it. 
Two principal methods are thus used to control spatial resolution quality of medical imaging systems. The results analysis of these methods can be automated. However, each method, even with automated analysis uses a physical test object.

\section{COMPARISON BETWEEN OTN AND OTP IN A TPS QUALITY ASSESSMENT}

Physical test objects are usually used to assess treatment planning systems quality. They are specially designed in a way to control each criterion and the TPS quality analysis is based on the knowledge of their shape. However, they require a scanner acquisition in order to be sent to the tested system. The geometry on which the result analysis is done is then deteriorated. Indeed, the scanner acquisition like every imaging system introduces essentially blur, noise, partial volume artefact and spatial distortions. The measures in the DRR are then marred by uncertainties and the quality control can not be precise. It is then done not only on the TPS but on the whole system including the scanner and the treatment planning system. A way to avoid this drawback of the physical phantom and the scanner errors introduction is to give perfect three dimensional volumes to the TPS. These perfectly known digital volumes are digital test objects (DTO).

In this section, a definition of digital test objects and their characteristics will firstly be presented. Secondly, a formal analysis of the quality control using physical and digital test objects will be done for each of the three quality criteria presented in the previous section. In each case, the quality measures of the two methods are compared.

\subsection{DTO definition}

A digital test object is a three dimensional matrix like a virtual model. It is used to replace the CT-data. The voxel values correspond to the density of the material that they represent. Like every test object, the geometry of the shapes included in the object is well known and it is designed in a way to control a specific quality criterion. Contrary to the physical test object, they do not need to go through a scanner acquisition. Thus their geometry is precise and not damaged by the scanner. The shapes constituting the DTO are designed for a specific test at the same time as the method to analyze the results is developed. That insures a consistency of the test and a possibility to compute a theoretical result.

The DTO are stored in a discrete format as well as in an analytic description. ${ }^{4}$ Each shape is described in a continuous way and have a discretization method that insures an equivalence between the two models. Thus only controlled manipulations are allowed in the shape creation to conserve this equivalence. This allows to modify easily the shapes dimensions, positions and orientations in the DTO in order to make the DTO adaptable to the system it controls. As a digital test object is not manufactured, it is much more adjustable than a physical test object. It is moreover easier to control the densities and the geometry of each shape. With a DTO the virtual model do not comport neither blur nor noise.

\subsection{Beam divergence quality control}

A digitally reconstructed radiograph (DRR) is an orthogonal projection of the virtual model onto a plane by the cast of a divergent beam. It is thus necessary to control the good divergence of the projection beam. A way to check it is to use a test object made up of two patterns that will be superimposed on the DRR if the followed divergence is correct. A bad divergence will be brought by a shifting of the source along the central ray axis.

In order to test this divergence quality test principle, a phantom made up a two rectangular patterns is proposed. The two rectangles are constituted by four thin segments. They are placed on two parallel planes, perpendicular to the central ray axis of the beam, one up and one down the isocenter. Their dimensions are chosen to correspond to the beam divergence. A diagram of this test object and its use is shown at figure 1 .

The divergence error can then be measured four times in each DRR image, once for each side of the projected rectangle. It is then possible to divide the image in four areas that are treated similarly (cf. figure 2). The distance between the projections of the two patterns is measured in each area. This distance is the parameter that allows a quantification of the TPS error in front of the DRR divergence quality criterion.

In a DTO case, it is easy to make some thin rectangular patterns by enlightenment of twos segments of rows and two segments of columns. With a null incidence angle for gantry, couch and collimator for the DRR 


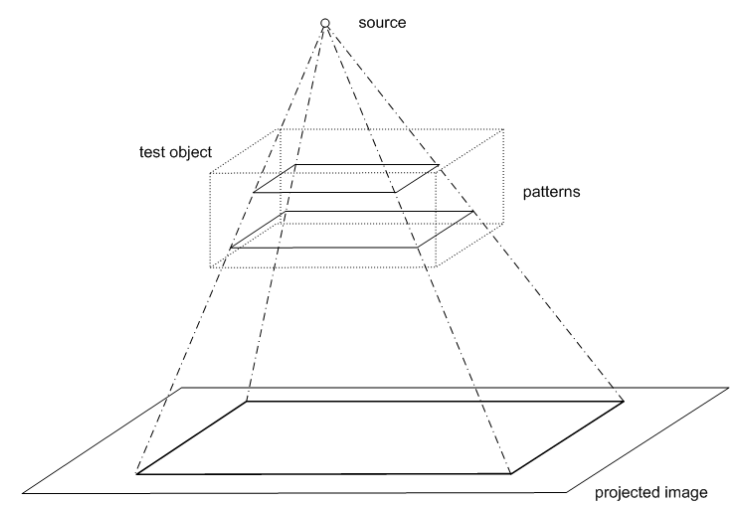

Figure 1. Diagram of the divergence test object.

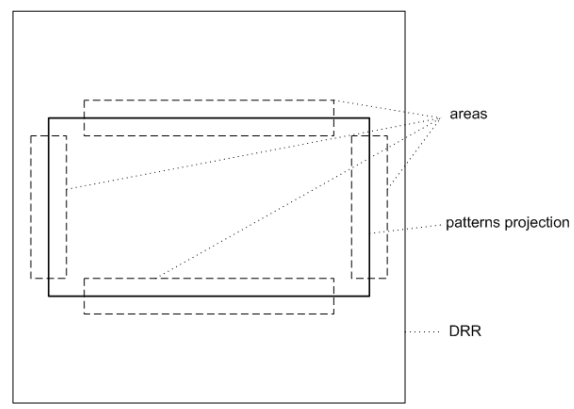

Figure 2. Diagram of the DRR division in four regions of interest.

computation, the projection of each side of the phantom pattern is perfectly aligned with the discrete grid of the image and the position of the two lines can be found by a projection of the area of the DRR (cf. figure $3(\mathrm{a})$ ). In a PTO case, the two patterns are created by the insertion of high density material thin wires in a low density test object. The PTO is positioned on the scanner table and aligned with the lasers before being scanned. The positioning of the object is done by the person who carries out the test and thus can not be perfect. Consequently, the patterns are not aligned with the discrete grid in the virtual model and then not aligned with the grid of the DRR image (cf. figure 3(b)). The projection of the image done to find the respective positions of the lines will not give any relevant information.

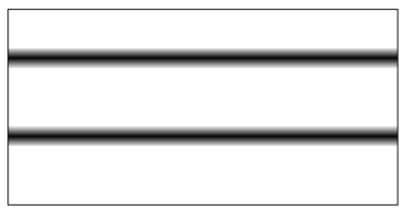

(a) DTO case.
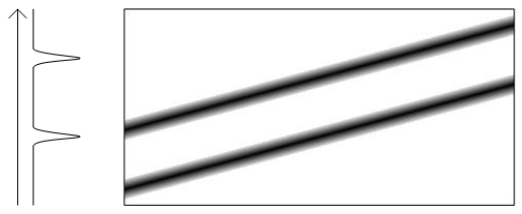

(b) PTO case.

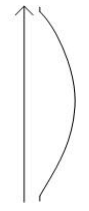

Figure 3. Diagrams of the projection of the divergence patterns in an area of the DRR, with a bad divergence.

A PTO result analysis requires then an additional step of image processing. Indeed, it is necessary to estimate the orientation of the lines ${ }^{17,18}$ in order to project them in the real orthogonal direction. This estimation step will present an error, that introduces an additional small bias in the divergence quality assessment.

Furthermore, a one voxel size segment in a digital test object is projected on several pixels of the DRR. The line that represents a pattern side is thus not thin but spread out and affects a few pixels. With the use of a PTO, the wires of the patterns are scanned and are represented by segments of several voxels large. Thus, the 
projection of these patterns are more spread on the DRR than with the use of a DTO. The consequence is that two close lines will not be distinguishable on the DRR because they are merged (cf. figure 4).

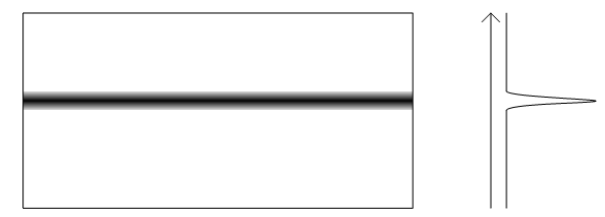

Figure 4. Diagram of the projection of the divergence patterns in an area of the DRR, with a perfect divergence.

With this divergence test method, it is essential to know from which distance it is possible to distinguish two lines. That will determine the smallest distinguishable error. In that purpose, it is possible to calculate the theoretical result that will be obtained if the divergence is correct i.e. if the two lines are superimposed. The principle is to calculate the orthogonal projection of the DRR image area of the two superimposed lines. For that purpose, it is necessary to know the projection of each line pattern individually and then to compute the superimposition result by summing the two obtained projections. It is thus easy to make two new DTOs identical to the first one but with only one rectangular pattern each, one with the upper pattern and the second with the lower pattern. The patterns can be exactly localized at the same position than in the complete DTO. A DRR of each of the new DTOs is computed as well as the orthogonal to the lines projection of the work area, and the theoretical result is obtained. In a PTO case, it is much more difficult to make identical test objects without one of the pattern and especially to scan them exactly in the same position as the first one. It is practically impossible to obtain the same virtual model but without one pattern. Thus the calculation of the theoretical result can not be done without errors. It thus can not be a real theoretical result. In order to evaluate the TPS DRR divergence error, the theoretical and the real results are compared. The smallest detectable error is determined by the capacity to see if there is a difference between the two results. The gaussian spread out of the pattern on the DRR is much more important in a PTO case and the theoretical result calculation is less accurate. Thus the use of a PTO will not allow a detection of such small errors as with DTO and implicates a less precise divergence quality assessment for the DRR computation.

\subsection{Beam incidence quality control}

The beam incidence control in DRR computation corresponds to check if the source of the virtual beam is well positioned. What is tested is not its distance from the isocenter, that corresponds to the divergence test, but its position toward the DTO volume position. The DRR is a beam eye view. A divergent beam is cast from a virtual source through the virtual 3D model of the patient or the test object and the projection of the different densities is calculated at the isocenter level. The image is perpendicular to the central ray of the beam. The isocenter is always projected in the center of the DRR image whatever the incidence is because it is located at the invariant point for the couch and gantry rotations. A point on the central ray is then cast onto the isocenter. If one of the voxel of the virtual model placed on the theoretical ray axis is lit, its projection will be superimposed to the isocenter projection if the incidence is correct and nearby it if the incidence is bad. The figure 5 illustrates this principle.

The quantifiable incidence error is represented by the angle $\theta$ which is the angle between the theoretical axis of the beam and the real axis of the beam. It can then be calculated by some trigonometric formulas (cf. figure 5 ). The first step to calculate $\theta$ is to calculate $\Delta$, the gap between isocenter and the projected point P. The error is only considered in one dimension for the calculations. From the knowing of $\Delta$ and the distance $D S I$ from source to the isocenter, $\theta^{\prime}$ can be calculated in the following way:

$$
\theta^{\prime}=\arctan \left(\frac{\Delta}{D S I}\right)
$$

The angle $\theta$ can then be calculated. As $\theta^{\prime}$ is acute and $D M I$, the distance from the lit voxel to the isocenter is 


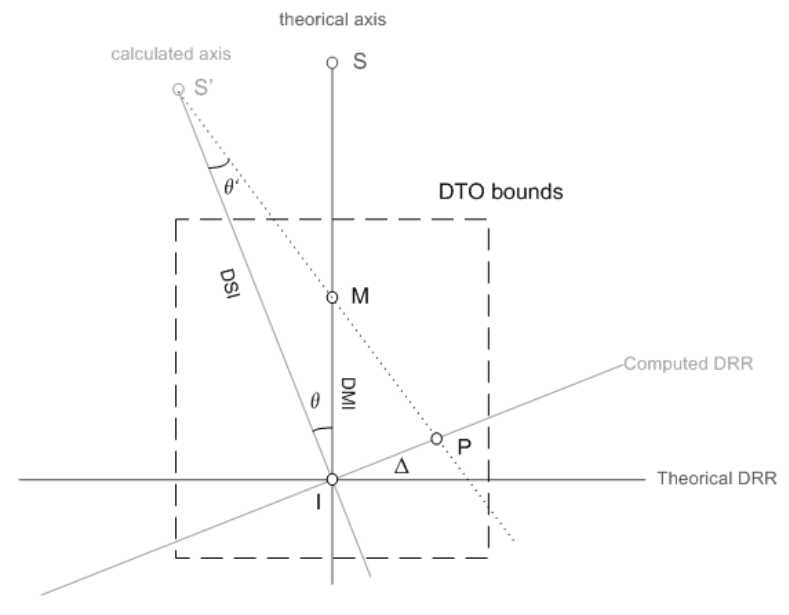

Figure 5. Diagram of DRR computation geometry. In dark gray are the theoretical beam axis (source S) and DRR, the ball pattern $\mathrm{M}$ is cast on the center of the image. In light gray are the real axis (source S') and DRR, M is cast on the DRR in the point P. $\Delta$ is the measured distance between the center of the image and the point $\mathrm{P}, D M I$ is the distance between $\mathrm{M}$ and the isocenter, DSI is the distance between the source and the isocenter and is the same for the real and the theoretical DRRs. $\theta^{\prime}$ is the angle between the beam axis and the ray going throw $\mathrm{S}^{\prime}$ and $\mathrm{M}$, and $\theta$ is the angle between the theoretical axis and the real one. In a perfect case, $\theta=0$.

inferior to $D S I$, it is possible to use the following formula:

$$
\theta=-\theta^{\prime}+\arcsin \left(\frac{D S I \times \sin \theta^{\prime}}{D M I}\right) .
$$

However, in order to validate this formula, the following condition has to be respected:

$$
D M I>D S I \times \sin \theta^{\prime} \quad \Leftrightarrow \quad \theta^{\prime}<\arcsin \left(\frac{D M I}{D S I}\right) .
$$

This condition is easily respected in the usual use of test objects and treatment planning systems, even with a big incidence error. Indeed, with a DSI equal to $1000 \mathrm{~mm}$ and a DSI equal to $100 \mathrm{~mm}$ for example, the maximum error that respects the previous condition is:

$$
\theta_{\text {max }}^{\prime}=\arcsin \left(\frac{D M I}{D S I}\right)=\arcsin \left(\frac{100}{1000}\right) \simeq 5,739
$$

from where $\theta_{\max } \simeq 39,26^{\circ}$. This maximum error is much superior to the error that can be presented by a TPS.

For this beam incidence control principle, it is necessary to determine the position of the point $P$ on the DRR and then to estimate the distance $I P$ from the isocenter to the projection. The point $P$ is materialized by the center of the dot corresponding to the projection of the lit voxel of the virtual model onto the DRR. In a first case in which a DTO is used, it is easy to create a virtual model with only one high density voxel, inside a global environment of low density. The position of the voxel in the DTO geometry is then perfectly known. The projection of this voxel is spread out on several pixels of the DRR image. The center of the voxel is projected on the center of the spot, that marks the point $P$. In a second case in which a similar PTO is used, the virtual model is computed from the acquisition of a physical phantom containing a ball made of high density material. Even if the ball is very small, its representation in the virtual model is blurred by the scanner acquisition and several voxels are affected by the ball representation. Thus the dispersion of the high density pattern onto the DRR is spread on more pixels than in the DTO case. The figure 6 illustrates this principle.

With this incidence quality test, the measured parameter that allows an error quantification is the vector constituted by distance $I P$ and the standard deviation on this distance:

$$
\text { param }=\left(I P, \sigma_{I P}\right)
$$




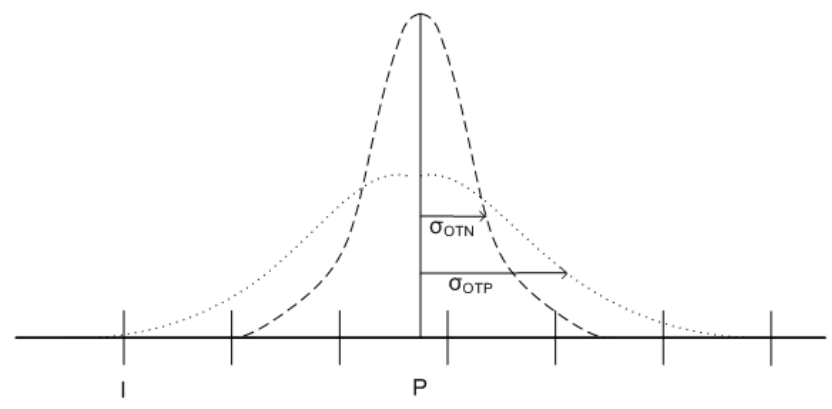

Figure 6. Diagram of the dispersion of the projection of a small high density pattern on a DRR in the DTO and PTO cases.

In the two cases the mean positions of $P$ and the mean $I P$ distances that can be measured are quite the same, the center of the spot will be projected at the same place. However, the standard deviations of the measures are very different for the two cases. It is much more important in the PTO case, as it can be seen in the figure 6 . Thus the measure of the distance $I P$ is more accurate in the DTO case, that implicates a more precise incidence quality assessment. If the spatial distortion that the scanner can introduce in the virtual model is taken into account in this calculation, it is obvious that the standard deviation on the distance IP for the PTO case will be higher.

\subsection{Spatial resolution quality control}

The spatial resolution quality assessment can be done with the help of a test object containing line pair patterns of different frequencies. As explained in the section 2.3, the MTF is then approached by the practical MTF calculated for each frequency the with Droege formula (cf. equation 1). DTO line pair patterns are built easily in the three dimensional grid of the discrete object affecting high density to chosen voxels. PTO line pair patterns are made of high density material parts included into a low density material box. The difference between the use of PTOs and DTOs is that PTOs need to be scanned and the CT-data is corrupted by the acquisition blur of the scanner. Thus the line pair patterns are not perfect in the virtual model. Altered high frequencies will not be correct on the DRR, even if the MTF of the DRR algorithm is high for this frequency. Indeed, the modulation transfer function that gives the spatial resolution of a system is the Fourier transform of the point spread function (PSF). The PSF of a system $P S F_{\text {system }}$ can simply be modeled by the convolution of a Dirac impulse $\Delta$ by the blur function $b f_{\text {system }}$ of the system:

$$
P S F_{\text {system }}=\Delta \otimes b f_{\text {system }} .
$$

In the case of the use of a DTO, the input is perfect and controlled, thus the computed PSF and MTF correspond to the ones of the DRR algorithm. In the case of the use of a PTO, the input is the result of a first blurring by the CT scanner. The PSF measured on the DRR is the convolution of the scanner PSF and the blur function of the DRR algorithm:

$$
P S F_{D R R}=\left(\Delta \otimes b f_{\text {scanner }}\right) \otimes b f_{D R R a l g o r i t h m},
$$

where $P S F_{D R R}$ is the PSF measured on the DRR, $\Delta$ is the Dirac function, $b f_{\text {scanner }}$ is the scanner blur function and $b f_{D R \text { Ralgorithm }}$ is the DRR algorithm PSF. Thus the MTF computed on a DRR for a given frequency with the help of a physical test object is not the DRR algorithm MTF but the whole system scanner plus DRR algorithm one:

$$
M T F_{\text {measured }}=T F\left(P S F_{\text {scanner }} \otimes P S F_{D R \text { Ralgorithm }}\right),
$$

where $M T F_{\text {measured }}$ is the computed MTF on the DRR, $T F$ is the Fourier transform, $P S F_{\text {scanner }}$ is the scanner PSF and $P S F_{D R R a l g o r i t h m}$ is the PSF of the DRR algorithm. That implicates that it will be impossible to compute a MTF on the DRR superior to the one of the scanner for the same frequency. The scanner MTF limits the quality assessment of the DRR algorithm. 


\section{DISCUSSION}

R. Wagner, D. Brown, K. Myers, H. Barrett and their young colleagues have popularized the generalized signal to noise ratio devoted to observers in the medical imaging community. As a matter of fact, this theory is perfectly adapted to characterize tasks for the quality control instead of using it only for the tasks defined from physician uses. ${ }^{19-22}$ According to its averaging over the frequencies spectrum, the SNR computed from the MTF (acquisition and algorithm), the power spectrum of the noise, and the task $\Delta S(\nu)$ represents the best way for assessing a given task of quality control as described in equation 11.

$$
S N R_{i^{2}}=\int \frac{[\Delta S(\nu)]^{2} \times G^{2} \times M T F^{2}(\nu)}{W(\nu)} d \nu
$$

This equation should be interpreted for both type of phantoms. As already said, the MTF algorithm factor is the same as well as the gain factor $G$. For the global MTF, it is obvious that the MTF of the scanner acquisition will degrade the high frequencies performances for the OTP case whereas the corresponding MTF is one for the OTN. The power spectrum of the noise $W(\nu)$ obeys the same rule: the acquisition generally gives a Poisson or Gaussian noise whereas almost no noise (except for sampling) will be observed recorded for the OTN.

At the opposite, the $\Delta S(\nu)$ will remain the same for both kind of phantoms. As exemplified in the paper, this equality only holds when the operator realizing the test is very careful while positioning the physical object. Otherwise, there is another degradation for the OTP case in the computation of the MTF.

As a result, the obtained SNR will automatically behave better for the OTN case.

The further separation of the SNR into the task and the NEQ representing the whole characterization of the device is also important here. This allows for obtaining the generic curve for the entire quality control procedure and then to apply it to each specific task of quality control. In our application, this NEQ can be written for the DRR and then DS specified for each individual task that have been presented.

Applying this theory is not only a simple way to enhance good practices for control quality. This gives a framework for specifying any quality control task and in our case to get observers that can prove the superiority of the OTN approach compared to the OTP ones.

\section{CONCLUSION}

The presented work leads to the definition of new methods to control automatically and precisely the quality of the DRR computation for the criteria of the ray divergence, ray incidence and spatial resolution. The new methods can take into account that the DRR obtained from the TPS depends on the user. These methods are very accurate, give quantitative results and are not affected by the CT scan defaults contrary to the classical methods.

Some tests have been carried out to compare TPS quality assessment with physical test objects and with digital test objects. Some DTOs were designed specially for each criterion as well as their automatic analysis method. The full comparison between DTO and PTO will use the framework of generalized SNR and NEQ

concepts used almost everywhere nowadays for assessing the correct dose a patient may have for a given medical imagery acquisition. Replacing the patient by the quality control phantom can then be simply view as an honest return over invest .

\section{REFERENCES}

1. T. Craig, D. Brochu, and J. Van Dyk, "A quality assurance phantom for three-dimensional radiation treatment planning," Int. J. Radiat. Oncol. Biol. Phys. 44(4), pp. 955-966, 1999.

2. K. McGee, I. Das, and C. Sims, "Evaluation of digitally reconstructed radiographs (DRRs) used for clinical radiotherapy: A phantom study," Med. Phys. 22, pp. 1815-1827, November 1995.

3. E. Denis, S. Beaumont, and J.-P. Guédon, "Digital reconstructed radiography quality control with software methods," in Proceedings of SPIE, Medical Imaging 2005: Physics of medical imaging, M. Flynn, ed., 5745, pp. 1002-1013, SPIE, 2005. 
4. E. Denis, J.-P. Guédon, S. Beaumont, and N. Normand, "Discrete and continuous description of a three dimensional scene for quality control of radiotherapy treatment planning systems," in Proceedings of SPIE, Medical Imaging 2006: Physics of medical imaging, M. Flynn, ed., 6142, SPIE, 2006.

5. N. A. Mason, The generation of a digital phantom for testing of digitally reconstructed radiographs. $\mathrm{PhD}$ thesis, University of South Florida, 2004.

6. S. Mutic, J. Palta, E. Butker, I. Das, M. Huq, L.-N. Dick Loo, B. Salter, C. McCollough, and J. Van Dyk, "Quality assurance for computed-tomography simulators and the computed-tomography-simulation process: Report of the AAPM Radiation Therapy Committee Task Group No. 66 ," Med. Phys. 30, pp. 2762-2792, October 2003.

7. B. Fraas, K. Doppke, M. Hunt, G. Kutcher, G. Starkschall, R. Stern, and J. Van Dyke, "American Association of Physicists in Medicine Radiation Therapy Committee Task Group 53: Quality assurance for clinical radiotherapy treatment planning," Med. Phys. 25, pp. 1773-1829, October 1998.

8. Modus, "Quality assurance system for advanced radiotherapy," Phys. Med. Biol. 47(9), pp. 1485-1492, 2002.

9. Modus Medical Devices Inc, Quasar multi-leaf collimator beam geometry phantom, User's Guide, 2003.

10. J.-N. Foulquier, "Contrôle de qualité d'une installation de simulation virtuelle. les outils de la simulation virtuelle," tech. rep., Hôpital Tenon, service de radiothérapie, unité de physique médicale, Paris, 2002.

11. J.-N. Foulquier and D. El-Babaa, H.and Lefkopoulos, "Fantôme pour le contrôle de qualité d'un système de simulation virtuelle d'un traitement de radiothérapie," 2004.

12. B. F. O'Connell, "Image Quality of Digitally Reconstructed Radiographs," tech. rep., University of Aberdeen, 2006.

13. A. J. Reilly, "Automated image analysis software for quality assurance of a radiotherapy ct simulator.".

14. R. T. Droege and R. L. Morin, "A parctical method to measure the mtf of ct scanners," Med. Phys. 9, pp. $758-760$, Sept/Oct 1982.

15. S. Kirwin and A. Langmack, "Determination of the modulation transfert function of digitally reconstructed radiographs in radiotherapy treatment planning using a point phantom," Phys. Med. Biol. 50, pp. N251N255, 2005.

16. A. J. Reilly, "Evaluation of AutoQALite image quality measurement software.".

17. F. Le Pouliquen, C. Germain, and P. Baylou, "Line orientation operator," in Proc. of IEEE - ICIP 2001, 3, pp. 708-711, IEEE, (Thessaloniki, Greece), October 2001.

18. J. Da Costa, F. Le Pouliquen, C. Germain, and P. Baylou, "New operators for optimized orientation estimation," in Proc. of IEEE - ICIP 2001, 3, pp. 744-747, IEEE, (Thessaloniki, Greece), October 2001.

19. R. F. Wagner and D. G. Brown, "Unified SNR analysis of medical imaging systems," Phys. Med. Biol. 30, pp. 489-518, 1985.

20. H. H. Barrett, J. L. Denny, R. F. Wagner, and K. J. Myers, "Objective assessment of image quality. ii. fisher information, fourier crosstalk, and figures of merit for task performance," J. Opt. Soc. Am. 12, pp. 834-852, 1995.

21. H. Barrett and K. Myers, Foundations of Image Science, Wiley, ed., 2004.

22. H. H. Barrett, C. K. Abbey, and E. Clarkson, "Objective assessment of image quality. iii. roc metrics, ideal observers, and likelihood generating functions," J. Opt. Soc. Am. 15, pp. 1520-1535, 1998. 